of Raffaele's findings by saying that they have not been confirmed or received any support. But it is just that negative assumption that needs support, a support that would exact a long painstaking work as was Raffaele's work.

I am sure that your readers may be surprised in learning from Davey that to succeed in finding these forms, with the same difficulties that one meets in finding them in a six-days-old chick, it would be necessary to inoculate in the human the contents of 50,000 infected salivary glands. All malariologists who took the trouble of looking at Raffaele's smears have not hesitated in considering them as belonging to the exo-erythrocytic stage. I think that the authors' findings in the monkey is a confirmation of Raffaele's work on man. By the way, owing to the authority of Raffaele in that field his observations should have been accepted

Before finishing this letter I feel the need of reminding your readers of the high intellectual merit of James in the discovery of the exo-erythrocytic stage. Considering all the proofs against Schaudinn's theory he postulated the necessity of a new stage between the sporozoite and the infection of the red blood cells. This working hypothesis was published in its completeness by Ruge (Dtsch. med. Wschr., 1936, 62, 1869) and has been proved true by successive work. I am glad to give homage to the memory of James as the malariologist to whom the experimental malaria owes the most interesting clinical results.-I am, etc.,

Istituto di Malariologia Ettore Marchiafava, Rome.

\section{G. Bastianelli.}

\section{Swine Erysipelas Infection in Man}

SIR,- I am interested in the concluding statement made by the president of the Section of Comparative Medicine of the Royal Society of Medicine (Feb. 28, p. 404) in which he asked whether the susceptibility of man to swine erysipelas might not be much higher than was commonly thought. Personally, I have found it comparatively common-its incidence twice as frequent as erysipelas in my practice. I meet at least two cases of the cutaneous type and sometimes as many as five of these cases in a year. I must have seen about fifty cases or more during the past eighteen years.

It is variously known as erysipeloid, crab cellulitis, or reticular lymphangitis. There is almost always a history of a scratch or minor cut from a meat bone or a skinning or butcher's knife. The infection has occurred in my cases among butchers, gamekeepers, cooks, farmers, who have been skinning dead sheep found on the hill, and not infrequently in housewives. A few have occurred from fish-bone scratches.

The condition has a seasonal incidence from August to February. It usually occurs on the finger, commencing as a purple patch, which extends over the finger, clearing in the centre and working towards the tip and the palm. There it commonly proceeds to travel down a neighbouring finger to its tip. It has a slowly spreading, sharply defined, purple, raised narrow margin. It is itchy and hot, but never really painful. On one occasion only have I seen a mild lymphangitis to the middle of the forearm. It resolved spontaneously with splinting of the parts. Suppuration never occurs. The erysipeloid undergoes spontaneous cure in about three weeks in most cases.

Until two years ago I treated these cases with $10 \%$ ichthyol ointment applied every two days on lint, knowing that it would at least do no harm. I have never seen a case relapse with this treatment although relapse js said to be common. I do not claim that the ichthyol had any particular effect on the infection, but some method of treatment had to be given to satisfy the patient. Lately I treated two cases with 200,000 units of penicillin night and morning, but abandoned it in both cases after five days as no improvement occurred. This autumn I was fortunate in having an elderly housewife with the condition, whose daughter, a nurse, administered 50,000 units of penicillin three-hourly for seven days. This case was less than a week old and too early to undergo spontaneous cure. After four days of penicillin treatment the lesion had completely disappeared, and no recurrence took place. It is obvious that ordinary massive dosage night and morning is insufficient, but 400,000 units daily by three-hourly injections effects cure in some cases. Higher dosage might of course be necessary in others.

I feel sure that swine erysipelas occurs much more frequently in man in rural areas than is commonly supposed.-I am, etc.,

\section{Rarer Manifestations of Herpes Zoster}

SIR,-The very interesting article on this subject by Dr. T Parkinson (Jan. 3, p. 8) is bound to recall other unusual cases of herpes zoster. I remember to have seen a sequence of cases a few years ago which struck me already at the time as extraordinary but gained even more significance after reading his article.

The first case was that of an elderly man, patient $\mathbf{A}$, who was admitted to hospital with herpes zoster of typical thoracic distribution. A few days later patient $B$ was admitted for an amputation of his toes. While recovering from it he used to visit patient $A$ in his room. About three weeks after his admission he developed a left facial palsy which took about one year before it had disappeared entirely. Patient A was admitted again several months later for post-herpetic pain and mental depression. At that time patient $C$. who was being treated for a diaphragmatic hernia with ulceration, visited him on several occasions. After about a fortnight he developed a herpes zoster of typical abdominal distribution. Somewhat about the same time patient $D$ was recovering from a haemorrhoidectomy. He too visited patient $\mathbf{A}$ now and then. Soon after his discharge he consulted me for a paralysis of the long thoracic nerve which had affected the serratus anterior muscle and the posterior portion of the deltoid.

These four cases are interesting inasmuch as two of them apparently were examples of "zoster sine herpete." They further seem to point to a carrier state in herpes zoster which possibly was present in patient A.

The relationship between herpes zoster and varicella was exemplified lately to me when I saw a man suffering from herpes zoster and his wife getting an attack of varicella about three weeks later.

Finally it is worth recording the case of a 6-year-old boy who had been under radium treatment for sarcoma after abdominal exploration and came to see me for a herpes zoster with a distribution corresponding with L5.-I am, etc.,

Gatton, Australia.

E. Guthaner.

\section{Treatment of Subacute Bacterial Endocarditis}

SIR,-I agree with much that Dr. Florence M. E. Davies (Feb. 14, p. 317) and Mr. B. Waters (Feb. 28, p. 416) have written on this subject. Nevertheless I do feel that the importance of infected teeth, apical granuloma, and possibly tonsils as potential sources of bacterial leakage leading to relapse has not been emphasized.

Therefore may $I$ add that I have shown elsewhere (Lancet. $1947,2,807)$ that penicillin-sensitive organisms can thrive in a dead tooth pulp in a subject receiving massive doses of penicillin.-I am, etc.,

Liverpool.

John Hallam.

\section{Breast-feeding in Erythroblastosis Foetalis}

SIR,-The practice of weaning infants with haemolytic disease because the mother's milk contains antibodies has been based on circumstantial evidence and speculation on the possible harm that might result to the baby rather than on scientific proof. The question of breast-feeding erythroblastic babies is of such importance that an authoritative opinion based upon actual investigation should be welcome. Dr. I. A. B. Cathie's observations (Oct. 25, 1947, p. 650) that there is no experimental evidence to prove that $\mathrm{Rh}$ antibodies are demonstrably absorbed into the blood stream by way of the alimentary canal and the conclusion that weaning of such infants is not justified are therefore of great interest. It is also interesting to note that at the Hospital for Sick Children, Great Ormond Street, London, it has been the practice to breast-feed such children whenever this can be done, irrespective of the antibody content of the breast milk, and that their clinical progress has not suggested any relationship between the antibodies in the milk and the duration of the haemolytic process.

I wish to make the following comments on these observations. The finding that $\mathrm{Rh}$ antibodies are not readily destroyed by the gastric secretion of infants is significant, as it shows that they remain potent and may yet be absorbed from the alimentary canal. There is a fundamental difference between absorption through the placenta and from the alimentary canal. In the former, on entry into the foetal circulation the antibodies come into direct contact with the 
foetal red cells which possess the specific antigen in the greatest concentration, and it is probable that most of the antibodies are specifically fixed to the red cells. Indeed the red cells may be said to constitute the first line of defence against the antibodies. In the latter, on the other hand, the antibodies have to pass through the liver, the cells of which have been shown by Boorman and Dodd (1943) to contain the $\mathrm{Rh}$ antigen, though not to the same extent as the erythrocytes. Is it possible that the antibodies are absorbed by the hepatic cells? If this view is correct it would seem reasonable to suppose that the antibodies can gain entrance into the circulation only after the liver cells have been more or less saturated with them.

What causes the lag in the recovery in the blood state of erythroblastic babies? Is it the haemolytic process or the hypoplasia of bone marrow, or both? The pathology of the haemolytic process in the infant may be compared with that of cases of blood transfusion from high-titre group $\mathbf{O}$ donors. Yet though the degree of haemolysis as judged by the fall in haemoglobin and red cell count may be severe it is but rarely attended with haemoglobinuria or evidence of renal damage such as oliguria and nitrogen retention. This is so even when Rh-positive blood has been used for transfusion. Clinically the jaundice may be minimal or there may be an increase if it was previously present. A rapidly increasing pallor is a common feature. These clinical findings suggest that the anaemia is probably also due to poor red cell regeneration rather than entirely to haemolysis.

It is well known that in animals physiological absorption of maternal antibodies via the alimentary canal is quite possible. The observations by Kerr and Robertson (1946) are of special interest in this connexion. Working with Trichomonas foetus antigen on cows these workers have shown that the antibodies against $T r$. foetus present in the whey of colostrum can be absorbed by the newborn calf from the first feed after birth-and be demonstrable within 2 hours after the feed. If the calf had been fed on boiled milk for 24 hours the antibody present in the colostrum is not absorbed. This shows that the conditions in the alimentary canal which permit the passage of the antibodies disappear within the first 24 hours. These workers have also shown that $\mathrm{Tr}$. foetus antibodies in serum can be absorbed via the alimentary canal into the blood stream in the newborn calf, though it is much less easily assimilated than from colostrum.

In this respect newborn calves and newborn babies appear to differ from each other. Parsons (1946) attributes this difference in mechanism " to the variation in placental structure ; in women the placenta is thin, the blood actually bathing the chorionic villi and thus rendering the transference easy, whereas in the cow it is thick." Several layers of tissue are interposed between the maternal and foetal circulations.

In spite of the lack of proof of antibody absorption by the mouth using the present methods, there is no reason why the experimental evidence in favour of absorption should not be advantageously applied to clinical medicine. As colostrum contains a higher concentration of $\mathrm{Rh}$ antibodies than breast milk it would only serve as an additional precaution if colostrum feeding is specially interdicted, though breast-feeding may be allowed as is the practice at the Hospital for Sick Children.-I am, etc.,

Madras, India.

K. S. Ranganathan.

REFERENCES

Boorman, K. E., and Dodd, B. E. (1943). J. Path. Bact., 55, 329

Kerr, W. R., and Robertson, M. (1946). J. comp. Path. Therap., 56, 101

Parsons, L. G. (1946). Canad. med. Ass. J., 65, 327.

\section{Natural Position for Childbirth}

SIR,-Dr. Kathleen Vaughan's letter (Jan. 31, p. 222) draws attention once more to the natural position for childbirth. Most of us must have had cases where a woman has delivered herself in this position. Nearly all women who are blessed by or who suffer from precipitate labour give birth when standing or squatting. often perhaps imagining that nothing more than movement of the bowels is taking place-that at any rate has been my experience. The most impressive demonstration that I have witnessed of the efficacy of the squatting position occurred in 1921. I had been called to a confinement at a lonely farmhouse, I believe the woman's eighth. My first visit was before 9 a.m. I called several times during the day and no progress was evident. She repeatedly proclaimed that she could never have the baby "on the bed," she must "get down to it." I called at 9 p.m. and found little or no change though the nurse appeared to be working hard with her in the left lateral position. She p!eaded with me to let her "squat," and so at my suggestion a horrified nurse spread a clean sheet on the floor, the patient squatted as illustrated by Dr. Vaughan, and in a few minutes the child was born easily and without damage to the perineum.

It has been my habit in forceps delivery to have the patient in the lithotomy position. This appears to me to be the easiest and most convenient position, especially when working with little or no skilled assistance, the thighs being held flexed on the abdomen by an "obstetric helper"-a strip of webbing passing behind the patient's neck and fastened just above each knee. In this position one has the nearest approach to natural squatting.

In her instructive and interesting book $\mathrm{Dr}$. Vaughan puts forth convincing arguments for more natural, and herefore more rational, habits of living, feeding, and methods of delivering-or allowing the woman to deliver herself-in the attempt to reduce maternal and neonatal deaths. She points out that sepsis figures largely in the Ministry of Health reports. This I think is very largely determined by the mother's diet and her resistance to infection. One of the worst cases of puerperal infection 1 attended, with pelvic abscess and peritonitis, occurred after the birth of a puny child with no manipulation or interference of any kind. This woman confessed to me afterwards that she had not carried out instructions for dieting or taking vitamins, but had starved herself to produce a small child and have an easy time. She had a very small babyand nearly died.

Not only is our management of pregnancies and confinements becoming more and more "scientific" and artificial, but so is the care of the newborn babe. We hear a greal deal of clinics, baby weighing, and dried milk, but very little of breast-feeding. That seems to be going out of fashion. Is the modern mother, well supplied with vitamins and priority milk, incanable of producing the proper food for her baby? On booking a case in the third month of pregnancy I was once asked, "And what is the best artificial food for the baby"? Recently 1 spent an hour in a news theatre where a modern British hospital was depicted with its up-tothe-minute maternity block, and flashed on the screen was a picture of a lusty baby vigorously sucking at his bottle. A far prettier and more suitable "shot" would have been a happy and contented mother with her baby at the breast. I recently met an Australian woman who had had two children in nursing homes in England. She said she was always a "slow beginner" with her milk, but after the third or fourth day always had an abundant supply; but she had to plead with her doctor and the staff at the homes not to put her babies on to artificial food before she had had a real try to feed them herself.

Doubtless a great number of stillbirths and neonatal deaths are due to disproportion and the consequent difficulties in delivery, but how many of these babier are wenklings ciue to deficiencies in the mother's diet? Especially before the days of the expectant mothers' priorities the percentage may have been high. This together with artificial feeding, pulmonary infections, and tight wrapping-as 1 have previously urgedmay account for many neonatal deaths. It is said that $50 \%$ of babies swaddled in the seventeenth century died in their first year. Even now, when expectant mothers receive priority foods, many of them may suffer from undernourishment, as they are unlikely to take the special supply of extras themselves if they have young children, who naturally become the beneficiaries.

I regret that Dr. Vaughan's Safe Childbirth. and Dr. G. Dick Read's Revelation of Childbirth were not available at the beginning of my career instead of at the end. I strongly recommend all who practise midwifery to study both these excellent books.-I am, etc.,

Huddersfield.

\section{S. H. WADDY.}

\section{Peptic Ulceration}

SiR,-With Mr. V. J. Kinsella's desire to apply Occam's Razor to clinico-pathological interpretations (Feb. 14. p. 318) I have full sympathy. He cites in analogy the chronic ulcer of the leg, an instance I have used for many years in this very connexion, but surely the production of pain in the peripheral nervous system has a mechanism different from that in the viscera? The middle term obtrudes itself without undue multiplication of the entities, particularly if we exclude for the purpose of this discussion extra-gastric causes such as pancreatic erosion. The interposition of the food relationshin of ulcer pain inevitably introduces gastric function as a middle term; the implication of the secretion as a cause is negatived bv a large number of considerations such as the absence of reaction to nure acidifiers and alkalizers $(\mathrm{HCl}$ and $\mathrm{NaOH})$, and the persistence of the same pain after the onset of a generalized obstructive mucosal gastritis with total achylia.

In carcinnma of the stomach the difficulty of explaining pain in the presence of achlorhydria was for long thought to be over- 